\title{
BMJ Open Dose-response associations between metabolic indexes and the risk of comorbid type 2 diabetes mellitus among rheumatoid arthritis patients from Northern China: a case- control study
}

Guangxiao Li, ${ }^{1}$ Weijun Chi, ${ }^{1}$ Bingqing Bai, ${ }^{2}$ Ying $\mathrm{Li}^{3}{ }^{3}$ Tingting Wei, ${ }^{2}$ Lingyu $\mathrm{Fu}^{1,2}$

To cite: Li G, Chi W, Bai B, et al. Dose-response associations between metabolic indexes and the risk of comorbid type 2 diabetes mellitus among rheumatoid arthritis patients from Northern China: a case-control study. BMJ Open 2019;9:e028011. doi:10.1136/ bmjopen-2018-028011

- Prepublication history and additional material for this paper are available online. To view these files, please visit the journal online (http://dx.doi org/10.1136/bmjopen-2018028011).

Received 18 November 2018 Revised 4 June 2019 Accepted 19 June 2019
Check for updates

(C) Author(s) (or their employer(s)) 2019. Re-use permitted under CC BY-NC. No commercial re-use. See rights and permissions. Published by BMJ.

For numbered affiliations see end of article.

Correspondence to

Professor Lingyu Fu; fulingyucmu@sina.com

\section{ABSTRACT}

Objective To investigate whether there were any differences in the patterns of metabolic abnormalities between patients with rheumatoid arthritis (RA) with comorbid type 2 diabetes mellitus (T2DM) and other populations, and to plot the doseresponse relationships between metabolic indexes and the risk of comorbid T2DM among patients with RA.

Design and setting This is a retrospective casecontrol study using electronic medical records (EMRs). Patients with RA and/or T2DM or controls who were admitted to the First Affiliated Hospital of China Medical University between April 2008 and December 2016 were retrospectively recruited through the EMR system. After age-matching and sex-matching, 261 controls, 274 patients with T2DM, 276 patients with $\mathrm{RA}$ and 151 patients with RA+T2DM were eventually recruited.

Results Patients with RA+T2DM exhibited higher levels of systolic blood pressure (SBP), fasting plasma glucose (FPG) and triglyceride (TG) than the RA only patients. Moreover, the proportions of impaired fasting glucose (IFG), and total cholesterol (TC) and low-density lipoprotein cholesterol (LDL-C) dyslipidaemia in the RA+T2DM group were higher than those in the RA alone group (for IFG: $28.48 \%$ vs $18.84 \%, p=0.02$; for TC: $25.17 \%$ vs $15.22 \%, p=0.01$; for LDL-C: $25.83 \%$ vs $17.03 \%$; $p=0.03$ ). Rheumatoid factor (RF) positivity and IFG were independent risk indicators for comorbid T2DM among patients with RA (for RF positivity: $\mathrm{OR}=0.45$; $95 \% \mathrm{Cl}: 0.29$ to $0.69 ; \mathrm{p}<0.001$; for IFG: $\mathrm{OR}=1.70 ; 95 \% \mathrm{Cl}: 1.04$ to $2.76 ; \mathrm{p}=0.03$ ).

Conclusion Linear dose-response associations between SBP, TC, TG and the risk of comorbid T2DM among patients with RA were observed, whereas a non-linear dose-response association between FPG and the risk of comorbid T2DM was found. Patients with RA+T2DM were more likely to exhibit metabolic abnormalities than RA only patients. Patients with RA+T2DM with metabolic abnormalities deserve more attention from rheumatologists and endocrinologists.

\section{INTRODUCTION}

Rheumatoid arthritis (RA), a systemic and chronic inflammatory disease characterised by

\section{Strengths and limitations of this study}

- Few studies have examined the metabolic abnormalities of rheumatoid arthritis (RA) and type 2 diabetes mellitus (T2DM) simultaneously, especially among the Northern Chinese population.

- Restricted cubic spline functions were applied to draw the dose-response curves that represent the associations between metabolic indexes and risk of comorbid T2DM among patients with RA.

- As the participants were recruited using electronic medical records and outpatient patients were not included, we were unable to calculate the exact incidence of T2DM in established patients with RA.

- The confounding effects that the between-group treatment differences have on metabolic indexes should not be neglected, though age- and sex-propensity score matching (PSM) was conducted.

joint swelling, joint pain and damage of synovial joints, may lead to functional disability and premature mortality. ${ }^{1}$ It was estimated that the prevalence of RA was approximately $0.24 \%$ worldwide, ${ }^{2}$ whereas the prevalence of RA could be as high as $1.02 \%$ in China. ${ }^{3}$ The prevalence of RA in the Northern Chinese population was slightly higher than that of the Southern Chinese population. ${ }^{4}$ Regardless of the great improvements in the treatment strategies for RA over the past decades, RA has caused a heavy disease burden globally, with a mortality rate of 0.8 per 100000 people. $^{2}$

Although the prevention of joint destruction during RA has been strongly emphasised in recent decades, there is now a high level of concern about the associated comorbidities among these patients. As reported in the COMORA study, patients with RA showed a higher prevalence of cardiovascular events, 
infections, osteoporosis and cancers compared with the general population. ${ }^{5}$ Furthermore, the number of comorbidities in patients with RA was positively related to disease activity and severity. ${ }^{6}$ Nevertheless, the management of those comorbidities is far from optimal. ${ }^{5}$

The risk of cardiovascular disease (CVD) in patients with RA is twofold higher than that of the general population. Moreover, evidence from a national database in the uric acid (UA) showed that type 2 diabetes mellitus (T2DM) was highly prevalent in patients with RA $(28.3 \%) .{ }^{7} \mathrm{RA}$ and T2DM share a common pathogenesis basis of inflammation, ${ }^{8}$ which has been hypothesised to play an important role in the development and propagation of atherosclerosis and CVD. ${ }^{9}$ The enhanced effects of RA on CVD risk were similar to those of T2DM. ${ }^{10-12}$ Therefore, patients with RA with comorbid T2DM (RA+T2DM) may suffer from a greater CVD burden.

Metabolic abnormalities are well-known CVD risk factors for both the RA and T2DM populations. A high prevalence of metabolic abnormalities will significantly increase the risk of CVD in RA, especially for those with comorbid T2DM. Therefore, the management of metabolic abnormalities is an urgent priority to reduce CVD risk in patients with RA+T2DM. Unfortunately, metabolic abnormalities in chronic disease are usually under poor control in China. One study from Chinese rural districts showed that the awareness, treatment and control of T2DM were as low as $60.11 \%, 54.8 \%$ and $18.77 \%$, respectively. ${ }^{13}$ Another multicentre study demonstrated a high prevalence of dyslipidaemia $(67.1 \%)$, but relatively low proportions of awareness, treatment and control in Chinese patients with T2DM $(68.7 \%, 55.9 \%$ and $39.5 \%$, respectively). ${ }^{14}$ Likewise, the management of hypertension is also suboptimal in Chinese patients with T2DM, with approximately $58.2 \%$ of the patients failed to achieve the recommended goal for blood pressure control. ${ }^{15}$ Meanwhile, a previous study showed that approximately $40 \%$ of patients with RA with CVD risk factors did not receive dyslipidaemia screening from their primary care providers. ${ }^{16}$ Therefore, a scientific management strategy for the risk factors for CVD in patients with chronic diseases remains a great challenge for both care providers and patients.

As the applications of electronic medical records (EMRs) increase, growing interest arises in the use of EMRs to facilitate medical research. ${ }^{17}$ EMRs have been adopted in previous studies to assist in T2DM or RA management in the USA. ${ }^{18} 19$ The current study was conducted to investigate whether there were any differences in the patterns of metabolic abnormalities between patients with RA+T2DM and other populations using EMRs. Additionally, as we were extremely interested in the continuous dose-response associations, restricted cubic spline (RCS) functions were applied to draw the dose-response curves that represent the associations between metabolic indexes and the risk of comorbid T2DM among patients with RA. Our study will provide evidence for integrated and intensive management for patients with RA+T2DM in a real-world clinical setting and will assist rheumatologists in managing CVD risk more effectively in this population.

\section{METHODS}

\section{Study design and patients}

The present study was designed as a hospital-based casecontrol study and was aimed at exploring the risk of developing T2DM in patients with RA. Patients with RA or T2DM admitted to the First Affiliated Hospital of China Medical University during April 2008 and December 2016 were retrospectively recruited from the EMR system. Patients who were referred to our inpatient department because of appendicitis were treated as the control group. Those controls were confirmed to have no history of T2DM, coronary artery disease, stroke, malignancies and inflammatory autoimmune disease after appropriate medical investigations. The EMRs were classified and coded according to the International Classification of Diseases-Tenth Revision (ICD-10). The codes for RA were M05.x-06.x, and codes for T2DM were E11.x, E13.x-14.x and the codes for appendicitis were K35.x-37.x.

Patients with RA or T2DM with a history of stroke or coronary artery disease (CAD) were excluded. All subjects under the age of 18 were also excluded. Patients with RA+T2DM were defined as those who developed T2DM on the basis of established RA. All of these patients should have been diagnosed RA first and then DM, which was reflected in the EMRs by a T2DM' duration shorter than the RA' duration. Otherwise, the patients were excluded from the current study. According to the inclusion and exclusion criteria, 477 patients with RA+T2DM, 4541 controls, 12824 patients with T2DM and 5502 patients with RA were recruited. After excluding the study subjects without some of the necessary indexes and using a propensity score matching (PSM) strategy, ${ }^{20} 151$ patients with RA+T2DM, 261 controls, 274 patients with T2DM and 276 patients with RA were eventually enrolled. The selection process for study participants is shown in the online supplemental figure 1 .

\section{Diagnosis criteria of T2DM and RA}

Study subjects would be diagnosed with T2DM if they met at least one of the following conditions ${ }^{8}$ : (1) previously diagnosed T2DM in the EMRs; (2) prescriptions for antidiabetic drugs; and (3) newly diagnosed T2DM according to laboratory tests that indicated a fasting plasma glucose $(\mathrm{FPG})>7.0 \mathrm{mmol} / \mathrm{L}$, a random glucose level $>11.1 \mathrm{mmol} / \mathrm{L}$, or a glucose tolerance test result $>11.1 \mathrm{mmol} / \mathrm{L}$. Patients who fulfilled the 2010 American College of Rheumatology (ACR)/EULAR or 1987 ACR classification criteria were diagnosed with RA. ${ }^{21} 22$

\section{Data collection}

The relevant data, including demographic characteristics, smoking and drinking habits, RA characteristics and medications used were extracted from the patients' EMRs. The $\mathrm{C}$ reactive protein (CRP) level, erythrocyte sedimentation rate (ESR), rheumatoid factor (RF) status 
and anticyclic peptide containing citrulline (anti-CCP) status were routinely examined in patients with RA but not in patients with T2DM and controls. Therefore, these indexes were unavailable in patients with T2DM and controls. Likewise, glycated haemoglobin (HbA1c) was only available in patients with T2DM and patients with RA+T2DM but not in patients with RA and controls. The laboratory tests were uniformly carried out by the laboratory centre of our hospital using overnight fasting venous blood samples.

\section{Definition}

A CRP $>10 \mathrm{mg} / \mathrm{L}$ or $\mathrm{ESR}>30 \mathrm{~mm} / \mathrm{h}$ were indicators of elevated inflammation markers. ${ }^{23} 24$ Hypertension was defined as systolic blood pressure (SBP) $>140 \mathrm{~mm} \mathrm{Hg}$ or diastolic blood pressure (DBP) $>90 \mathrm{~mm} \mathrm{Hg}$. If the FPG was ranged from 5.6 to $6.9 \mathrm{mmol} / \mathrm{L}$, the patients were classified as having impaired fasting glucose (IFG). Hyperuricaemia was defined as a serum UA level $>0.42 \mathrm{mmol} / \mathrm{L}$ for men and postmenopausal women or $>0.36 \mathrm{mmol} / \mathrm{L}$ for premenopausal women. ${ }^{8}$ Dyslipidaemia was diagnosed according to the newly revised version of guidelines on the prevention and treatment of dyslipidaemia in Chinese adults. ${ }^{25}$ The proposed cutoff values for total cholesterol (TC), triglycerides (TG), high-density lipoprotein cholesterol (HDL-C) and low-density lipoprotein cholesterol (LDL-C) were 5.2, 1.7, 1.0 and $3.4 \mathrm{mmol} / \mathrm{L}$, respectively. The cutoff point for the TC/HDL-C ratio was set at $4.5 .^{26}$

\section{Statistical analysis}

Continuous variables were expressed as the mean $\pm \mathrm{SD}$ or medians with interquartile range (25th, 75 th), as appropriate. Categorical variables were expressed as frequencies (percentage). One-way analysis of variance was used to examine differences in the mean of continuous variables, followed by post hoc tests using the Fisher least-significant difference strategy where appropriate. The Wilcoxon rank-sum test was used to compare differences in medians. The $\chi^{2}$ tests were used to compare the proportions of categorical variables. Univariate and multivariate logistic regression analyses were sequentially conducted to further assess the associations between inflammatory markers, metabolic indexes and risk of T2DM among patients with RA, and the OR with the corresponding 95\% CI was also calculated. The candidate variables for multivariate logistic regression analyses were those with $\mathrm{p} \leq 0.10$ after the univariate logistic regression analyses.

As RCS makes it possible to characterise flexible and visible dose-response relationships between an exposure and outcome, ${ }^{27}$ this method was used to depict the dose-response associations between continuous variables and the risk of T2DM in established patients with RA based on logistic regression models. The adjusted covariates included age, sex and other potential confounders. The reference values were set at $140 \mathrm{~mm} \mathrm{Hg}$ for SBP, $90 \mathrm{~mm} \mathrm{Hg}$ for DBP, $6.1 \mathrm{mmol} / \mathrm{L}$ for FPG, $5.2 \mathrm{mmol} / \mathrm{L}$ for TC, $1.7 \mathrm{mmol} / \mathrm{L}$ for TG, $10 \mathrm{mg} / \mathrm{L}$ for CRP, $1.0 \mathrm{mmol} / \mathrm{L}$ for HDL-C, $3.4 \mathrm{mmol} / \mathrm{L}$ for LDL-C, 4.5 for the TC/
HDL-C ratio and $0.36 \mathrm{mmol} / \mathrm{L}$ for UA. The number of commonly used spline knots could be 3 (5th, 50th and 95th percentiles), 4 (5th, 25th, 75 th and 95 th percentiles) or 5 (5th,10th, 50th, 90th and 95th percentiles), and was finally determined by the Akaike information criterion (AIC), a statistical index to evaluate model goodness of fit, where the smaller the value is the better. ${ }^{28}$ The SAS macro was provided by Desquilbet and Mariotti, ${ }^{28}$ and had been previously used in an article by our group. ${ }^{29}$ The SAS macro provided tests for both overall and non-linear associations between risk indicators and the risk of concomitant T2DM among patients with RA. If the test for an overall association between indicator and risk of T2DM was statistically significant, the indicator was significantly associated with T2DM, regardless of the shape of the associations. Furthermore, if the test for the non-linear associations was also statistically significant, the association was significantly not linear.

PSM was performed using SPSS software (V.22.0, IBM Corporation, Armonk, New York, USA). ${ }^{30}$ The matching factors were age and sex, and the matching ratio was 1:2:2:2 for RA+T2DM, controls, T2DM alone and RA only subjects, respectively. Logistic regression models were applied to estimate the propensity scores, and the participants were matched using the nearest neighbour matching algorithm with a calliper width of $0.05 .{ }^{31}$ The other statistical analyses were conducted using SAS software (V.9.3). All tests were two-tailed, with a p value $<0.05$ considered statistically significant.

\section{Patient and public involvement}

The patients were not actively involved during the design and conduct of this study. The patients and the general public will be informed of the study results through peer-reviewed journals.

\section{RESULTS}

\section{Basic characteristics}

As shown in table 1, there were 151 patients with RA+T2DM (33 men and 118 women), with a mean age of $61.00 \pm 9.53$ years. The percentages of current tobacco and alcohol consumers in these patients were $8.61 \%$ and $3.97 \%$, respectively. No significant differences were observed in mean age, sex and proportions of smoking and alcohol consumption among the four groups of study subjects $(\mathrm{p}>0.05$ for all).

The median duration of T2DM in the RA+T2DM group was much lower than that of the T2DM group (18 vs 78 months, $\mathrm{p}<0.001$ ). There was no significant difference in the median RA duration between the RA+T2DM and RA groups (24 vs 36 months, $\mathrm{p}=0.69$ ). Likewise, our results showed no differences in the mean CRP and ESR levels between the RA+T2DM and RA groups (for CRP: 36.70 vs $36.19 \mathrm{mg} / \mathrm{L}, \mathrm{p}=0.92$; for ESR: $38.46 \mathrm{vs} 41.88 \mathrm{~mm} / \mathrm{h}, \mathrm{p}=0.40)$. The proportions of the anti-CCP-positive patients were similar between the $\mathrm{RA}+\mathrm{T} 2 \mathrm{DM}$ and RA groups $(46.00 \%$ vs $53.99 \%, \mathrm{p}=0.17)$. 
Table 1 The basic characteristics of different group of participants

\begin{tabular}{|c|c|c|c|c|c|}
\hline Characteristic & $\begin{array}{l}\text { Controls } \\
(n=261)\end{array}$ & $\begin{array}{l}\text { T2DM } \\
(n=274)\end{array}$ & $\begin{array}{l}\text { RA } \\
(n=276)\end{array}$ & $\begin{array}{l}\text { RA+T2DM } \\
(n=151)\end{array}$ & $P$ value \\
\hline Age (years) & $60.22 \pm 11.99$ & $60.85 \pm 9.34$ & $61.10 \pm 9.26$ & $61.00 \pm 9.53$ & 0.77 \\
\hline Current smoker (\%) & $17(6.51)$ & $21(7.66)$ & $21(7.64)$ & $13(8.61)$ & 0.88 \\
\hline Alcohol consumption (\%) & $13(4.98)$ & $15(5.47)$ & $14(5.07)$ & $6(3.97)$ & 0.93 \\
\hline \multicolumn{6}{|l|}{ Duration of RA (months) } \\
\hline Median (25th, 75th) & NA & NA & $36(6 \sim 120)$ & $24(4 \sim 120)$ & 0.69 \\
\hline CRP (mg/L) & NA & NA & $36.19 \pm 49.55$ & $36.70 \pm 53.37$ & 0.92 \\
\hline $\operatorname{ESR}(\mathrm{mm} / \mathrm{h})^{*}$ & NA & NA & $41.88 \pm 31.86$ & $38.46 \pm 29.20$ & 0.40 \\
\hline Traditional DMARDs (\%) & NA & NA & $210(76.09)$ & $120(79.47)$ & 0.43 \\
\hline Biologic DMARDs (\%) & NA & NA & $24(8.70)$ & $19(12.58)$ & 0.20 \\
\hline Oral antidiabetic drugs (\%) & NA & $119(43.43)$ & NA & $115(76.16)$ & $<0.001$ \\
\hline Insulin (\%) & NA & $94(34.31)$ & NA & $64(42.38)$ & 0.10 \\
\hline Statin (\%) & NA & 69 (25.18)† & $21(7.61)^{\star \star \star}$ & $36(23.84)$ & $<0.001$ \\
\hline Antihypertensive drugs (\%) & NA & $76(27.74)^{\star \star \star}$ & $68(24.64)^{\star \star \star}$ & 77 (50.99) & $<0.001$ \\
\hline
\end{tabular}

${ }^{*}$ ESR and anti-CCP level were unavailable in 77 and 51 patients with RA+T2DM, respectively.

${ }^{* * *} P$ value $<0.001$ when compared with the $R A+T 2 D M$ group.

†P value $>0.05$ when compared with the RA+T2DM group.

Bold text indicated that the overall analysis across groups was statistically significant.

Anti-CCP, anticyclic peptide containing citrulline; CRP, C reactive protein; DMARDs, disease-modifying antirheumatic drugs; ESR, erythrocyte sedimentation rate; F, female; M, male; NA, not available; RA, rheumatoid arthritis; RF, rheumatoid factor; T2DM, type 2 diabetes mellitus.

\begin{tabular}{|c|c|c|c|c|c|}
\hline Characteristic & $\begin{array}{l}\text { Controls } \\
(n=261)\end{array}$ & $\begin{array}{l}\text { T2DM } \\
(n=274)\end{array}$ & $\begin{array}{l}\text { RA } \\
(n=276)\end{array}$ & $\begin{array}{l}\text { RA+T2DM } \\
(\mathrm{n}=151)\end{array}$ & $P$ value \\
\hline SBP (mm Hg) & $126.79 \pm 18.63^{* * *}$ & $135.51 \pm 19.09 \dagger$ & $128.82 \pm 19.60^{* *}$ & $134.35 \pm 19.73$ & $<0.001$ \\
\hline $\mathrm{DBP}(\mathrm{mm} \mathrm{Hg})$ & $74.21 \pm 11.97^{\star \star}$ & $81.46 \pm 10.69^{\star \star}$ & $76.44 \pm 11.63 \dagger$ & $77.79 \pm 11.14$ & $<0.001$ \\
\hline FPG (mmol/L) & $5.47 \pm 0.98^{* \star *}$ & $9.78 \pm 4.85^{\star \star \star}$ & $5.25 \pm 0.90^{\star \star \star}$ & $7.04 \pm 2.14$ & $<0.001$ \\
\hline HbA1c (\%) & NA & $8.30 \pm 2.37^{\star \star \star}$ & NA & $7.24 \pm 1.56$ & $<0.001$ \\
\hline $\mathrm{TC}(\mathrm{mmol} / \mathrm{L})$ & $4.70 \pm 1.15 \dagger$ & $5.00 \pm 1.29^{\star \star}$ & $4.29 \pm 1.02 \dagger$ & $4.55 \pm 1.26$ & $<0.001$ \\
\hline TG (mmol/L) & $1.30 \pm 0.97 \dagger$ & $2.18 \pm 2.76^{\star \star \star}$ & $1.27 \pm 0.82^{*}$ & $1.63 \pm 1.88$ & $<0.001$ \\
\hline $\mathrm{HDL}-\mathrm{C}(\mathrm{mmol} / \mathrm{L})$ & $1.24 \pm 0.39^{\star \star \star}$ & $1.20 \pm 0.38^{\star \star}$ & $1.08 \pm 0.34 \dagger$ & $1.10 \pm 0.36$ & $<0.001$ \\
\hline LDL-C (mmol/L) & $2.95 \pm 0.98 \dagger$ & $3.11 \pm 0.99^{\star \star}$ & $2.70 \pm 0.86 \dagger$ & $2.79 \pm 0.92$ & $<0.001$ \\
\hline TC/HDL-C & $4.23 \pm 2.34 \dagger$ & $4.47 \pm 1.48 \dagger$ & $4.24 \pm 1.29 \dagger$ & $4.46 \pm 1.55$ & 0.22 \\
\hline UA (mmol/L) & $0.29 \pm 0.09 \dagger$ & $0.30 \pm 0.10 \dagger$ & $0.26 \pm 0.09 \dagger$ & $0.28 \pm 0.11$ & $<0.001$ \\
\hline
\end{tabular}

${ }^{*} \mathrm{P}$ value $<0.05$ when compared with the $\mathrm{RA}+\mathrm{T} 2 \mathrm{DM}$ group.

${ }^{*} \mathrm{P}$ value $<0.01$ when compared with the RA+T2DM group.

${ }^{* * *} \mathrm{P}$ value $<0.001$ when compared with the $\mathrm{RA}+\mathrm{T} 2 \mathrm{DM}$ group.

†P value $>0.05$ when compared with the RA+T2DM group.

DBP, diastolic blood pressure; FPG, fasting plasma glucose; HbA1c, glycated haemoglobin; HDL-C, high-density lipoprotein cholesterol; LDL-C, low-density lipoprotein cholesterol; NA, not available; RA, rheumatoid arthritis; SBP, systolic blood pressure; T2DM, type 2 diabetes mellitus; TC, total cholesterol; TG, triglycerides; UA, uric acid. 
A total of $58.28 \%$ of the patients in the RA+T2DM group were RF positive, which was significantly lower than the $75.36 \%$ in the RA group $(\mathrm{p}<0.001)$. In contrast, the proportion of patients taking corticosteroids in the RA+T2DM group was higher than that in the RA group $(76.82 \%$ vs $60.87 \%, \mathrm{p}<0.001)$. The usage of traditional disease-modifying antirheumatic drugs (DMARDs) and biologic DMARDs did not significantly differ between the $\mathrm{RA}+\mathrm{T} 2 \mathrm{DM}$ and $\mathrm{RA}$ groups $(\mathrm{p}=0.43$ and 0.20 , respectively). Our results indicated that patients in the RA+T2DM group were more likely to take oral antidiabetic drugs than those in the T2DM group $(76.16 \%$ vs $43.43 \%, \mathrm{p}<0.001)$, but the use of insulin injections was comparable between the two groups of patients $(\mathrm{p}=0.10)$. The percentages of statin use among the patients with T2DM, RA and RA+T2DM were $25.18 \%$, $7.61 \%$ and $23.84 \%$, respectively $(23.84 \%$ vs $25.18 \%$, $\mathrm{p}=0.76 ; 23.84 \%$ vs $7.61 \%, \mathrm{p}<0.0001)$. The percentages of antihypertensive drug use in the patients with T2DM, RA and RA+T2DM were $27.74 \%, 24.64 \%$ and $50.99 \%$, respectively.

\section{Comparison of metabolic parameters between different groups of participants}

As presented in table 2, SBP in the RA+T2DM group was obviously higher than that in the RA and control groups $(\mathrm{p}<0.01$ and $<0.001$, respectively). DBP in the RA+T2DM group was significantly lower than that in the T2DM group $(\mathrm{p}<0.01)$, but higher than that in the control group $(p<0.01)$. FPG concentrations in the patients with RA+T2DM were significantly higher than those in the controls $(\mathrm{p}<0.001)$ and patients with RA $(p<0.001)$, but significantly lower than those in the patients with T2DM $(\mathrm{p}<0.001)$. The HbAlc concentrations in the RA+T2DM group were significantly lower than those in the T2DM group $(\mathrm{p}<0.001)$.

As for plasma lipids, the TC, TG, HDL-C and LDL-C concentrations in the RA+T2DM group were lower than those in the T2DM group $(\mathrm{p}<0.01,<0.001,<0.01$ and $<0.01$, respectively). TG concentrations in the patients with RA+T2DM were significantly higher than those in the patients with RA $(p<0.05)$. Conversely, HDL-C concentrations in the patients with RA+T2DM were significantly lower than those in the controls. However, the plasma UA levels and TC/HDL-C ratio in the RA+T2DM group were not significantly different from those in the other three groups.

Comparison of elevated inflammation markers and metabolic abnormalities between patients with RA with and without comorbid T2DM

The previously mentioned cutoff values for the different indexes were used to calculate the percentages of metabolic abnormalities in the RA and RA+T2DM groups, and then chi-square tests were applied to examine their differences between groups. No significant differences were observed in the percentages of patients with elevated CRP and ESR between the two groups. Approximately half of the patients with RA+T2DM had an HbA1c level $>7 \%$ $(47.68 \%)$. The proportion of patients with IFG in the RA+T2DM group was obviously higher than that in the RA group $(28.48 \%$ vs $18.84 \%, \mathrm{p}=0.02)$. Plasma TC and LDL-C dyslipidaemia were more prevalent in the RA+T2DM group than in the RA group (for TC: $25.17 \%$ vs $15.22 \%$, $\mathrm{p}=0.01$; for LDL-C: $25.83 \%$ vs $17.03 \%, \mathrm{p}=0.03$ ). The incidences of hypertension, TG, HDL-C, hyperuricaemia as

Table 3 Comparison of elevated inflammation markers and metabolic abnormalities between patients with RA with and without comorbid T2DM

\begin{tabular}{|c|c|c|c|c|}
\hline Variables & RA (\%) & RA+T2DM (\%) & $\chi^{2}$ & $P$ value \\
\hline $\mathrm{CRP}>10 \mathrm{mg} / \mathrm{L}$ & $171(61.96)$ & 81 (53.64) & 2.79 & 0.09 \\
\hline $\mathrm{ESR}>30 \mathrm{~mm} / \mathrm{h}^{\star}$ & $147(53.26)$ & $39(52.70)$ & 0.01 & 0.93 \\
\hline $\mathrm{HbA} 1 \mathrm{c} \geq 7 \%$ & NA & $72(47.68)$ & NA & NA \\
\hline IFG & $52(18.84)$ & $43(28.48)$ & 5.24 & 0.02 \\
\hline Hypertension & $91(32.97)$ & $62(41.06)$ & 2.78 & 0.10 \\
\hline \multicolumn{5}{|l|}{ Dyslipidaemia } \\
\hline $\mathrm{TC}>5.2 \mathrm{mmol} / \mathrm{L}$ & $42(15.22)$ & $38(25.17)$ & 6.34 & 0.01 \\
\hline $\mathrm{TG}>1.7 \mathrm{mmol} / \mathrm{L}$ & 55 (19.93) & $41(27.15)$ & 2.92 & 0.09 \\
\hline $\mathrm{HDL}-\mathrm{C}<1.0 \mathrm{mmol} / \mathrm{L}$ & $121(43.84)$ & $72(47.68)$ & 0.58 & 0.45 \\
\hline $\mathrm{LDL}-\mathrm{C}>3.4 \mathrm{mmol} / \mathrm{L}$ & 47 (17.03) & $39(25.83)$ & 4.70 & 0.03 \\
\hline TC/HDL-C >4.5 & $95(34.42)$ & 63 (41.72) & 2.23 & 0.14 \\
\hline Hyperuricaemia & $17(6.16)$ & $11(7.28)$ & 0.20 & 0.65 \\
\hline
\end{tabular}

${ }^{*} \mathrm{ESR}$ level is not available in 77 patients with RA+T2DM.

Bold text indicated that the Chi-square test between groups was statistically significant.

CRP, C reactive protein; ESR, erythrocyte sedimentation rate; HbA1c, glycated haemoglobin; HDL-C, high-density lipoprotein cholesterol; IFG, impaired fasting glucose; LDL-C, low-density lipoprotein cholesterol; RA, rheumatoid arthritis; T2DM, type 2 diabetes mellitus; TC, total cholesterol; TG, triglycerides. 
Table 4 The logistic regression analysis for the risk of comorbid T2DM among patients with RA

\begin{tabular}{|c|c|c|c|c|c|c|}
\hline \multirow[b]{2}{*}{ Variables } & \multicolumn{3}{|l|}{ Univariate } & \multicolumn{3}{|l|}{ Multivariate } \\
\hline & Crude OR & $95 \% \mathrm{Cl}$ & $P$ value & Adjusted OR* & $95 \% \mathrm{Cl}$ & $P$ value \\
\hline $\mathrm{CRP}>10 \mathrm{mg} / \mathrm{L}$ & 0.71 & 0.48 to 1.06 & 0.10 & 0.71 & 0.46 to 1.10 & 0.13 \\
\hline IFG & 1.72 & 1.08 to 2.73 & 0.02 & 1.70 & 1.04 to 2.76 & 0.03 \\
\hline Hypertension & 1.42 & 0.94 to 2.13 & 0.10 & 1.32 & 0.86 to 2.03 & 0.20 \\
\hline TG dyslipidaemia & 1.50 & 0.94 to 2.38 & 0.09 & 1.23 & 0.74 to 2.06 & 0.42 \\
\hline LDL-C dyslipidaemia & 1.70 & 1.05 to 2.74 & 0.03 & 1.11 & 0.54 to 2.25 & 0.78 \\
\hline
\end{tabular}

${ }^{*}$ Age and sex served as additional adjusted factors.

Bold text indicated that multivariate logistic regression analysis for the variable was statistically significant.

CRP, C reactive protein; IFG, impaired fasting glucose; LDL-C, low-density lipoprotein cholesterol; RA, rheumatoid arthritis; RF, rheumatoid factor; T2DM, type 2 diabetes mellitus; TC, total cholesterol; TG, triglycerides.

well as the TC/HDL-C ratio were comparable between the two groups (table 3 ).

\section{Association between elevated inflammation markers, metabolic abnormalities and risk of comorbid T2DM among patients with RA}

Multivariate logistic regression analysis showed that RF positivity and IFG were independent risk factors for comorbid T2DM among patients with RA. RF positivity was significantly associated with a $55 \%$ reduction $(\mathrm{OR}=0.45$; 95\% CI: 0.29 to 0.69 ) in T2DM risk among patients with RA. In contrast, IFG was significantly associated with a 1.70-fold increase (OR=1.70;95\% CI: 1.04 to 2.76) in comorbid T2DM risk among patients with RA (table 4). We did not find any significant association between the other indexes and the risk of comorbid T2DM among patients with RA.

\section{Dose-response analyses of the associations between inflammation markers, metabolic indexes and risks of comorbid T2DM among patients with RA}

As shown in figure 1A, the dose-response curve indicated a linear association between SBP and the risk of comorbid T2DM among patients with RA (test for overall association: $\mathrm{p}=0.02$; test for non-linear association: $\mathrm{p}=0.71 ; \mathrm{AIC}=542.17$ ). Similarly, statistically significant linear dose-response associations between TC, TG and risk of comorbid T2DM were observed (for TC, test for the overall association: $\mathrm{p}=0.03$; test for non-linear association: $\mathrm{p}=0.20, \mathrm{AIC}=542.88$; for $\mathrm{TG}$, test for overall association: $\mathrm{p}=0.05$, test for the non-linear association: $\mathrm{p}=0.75, \mathrm{AIC}=542.13$ ) (figure $2 \mathrm{~A}$ and $\mathrm{B}$ ). In contrast, the dose-response association between FPG and the risk of comorbid T2DM was found to be non-linear (test for the overall association: $\mathrm{p}<0.001$, test for the non-linear association: $\mathrm{p}=0.04, \mathrm{AIC}=480.01$ ) (figure 1B). However, our results failed to show any significant dose-response associations between CRP, DBP, HDL-C, LDL-C, the TC/ HDL-C ratio, UA and risk of comorbid T2DM among patients with RA (online supplemental figures 2-4).

\section{Risk predictions based on the constructed dose-response curves}

Risk predictions were performed using the doseresponse curves previously constructed. As shown in the online supplemental tables 1-4, the ORs and their corresponding 95\% CIs for any given SBP, FPG, TC and TG values within the specified variation ranges could be obtained from those dose-response curves, with a group of specific values serving as references $(140 \mathrm{~mm} \mathrm{Hg}$ for SBP, $6.1 \mathrm{mmol} / \mathrm{L}$ for FPG, $5.2 \mathrm{mmol} / \mathrm{L}$ for TC and $1.7 \mathrm{mmol} / \mathrm{L}$ for TG). For instance, patients with RA with a TC level of $5.7 \mathrm{mmol} / \mathrm{L}$ had a 1.16 -fold (OR=1.16; $95 \%$ CI: 1.01 to 1.33) higher risk for comorbid T2DM than those with a TC level of $5.2 \mathrm{mmol} / \mathrm{L}$. Conversely, patients with RA with a TC level of $3.7 \mathrm{mmol} / \mathrm{L}$ were $46 \%$ (OR=0.54; $95 \%$ CI: 0.31 to 0.92 ) less likely to suffer from comorbid T2DM, than those with a reference level TC.

\section{DISCUSSION}

To the best of our knowledge, few studies have examined the metabolic abnormalities of RA and T2DM simultaneously, ${ }^{8}$ especially among the Northern Chinese population. Our findings showed that patients with RA+T2DM exhibited higher levels of SBP, FPG and TG than the RA only patients, but had lower levels of DBP, FPG, HbAlc, TC, TG, HDL-C and LDL-C than the T2DM only patients. Compared with controls, patients with RA+T2DM had higher levels of SBP, DBP and FPG but relatively lower levels of HDL-C. Moreover, the percentages of patients with IFG, and TC and LDL-C dyslipidaemia in the RA+T2DM group were significantly higher than those in RA only group. RF positivity and IFG were independent risk factors for comorbid T2DM among patients with RA. We speculated that the relatively lower proportion of RF-positive patients in the RA+T2DM group might partly be attributed to the anti-inflammatory effect of some oral antidiabetic drugs such as metformin. ${ }^{32} 33$ Interestingly, the dose-response associations between SBP, TC, TG and 
A

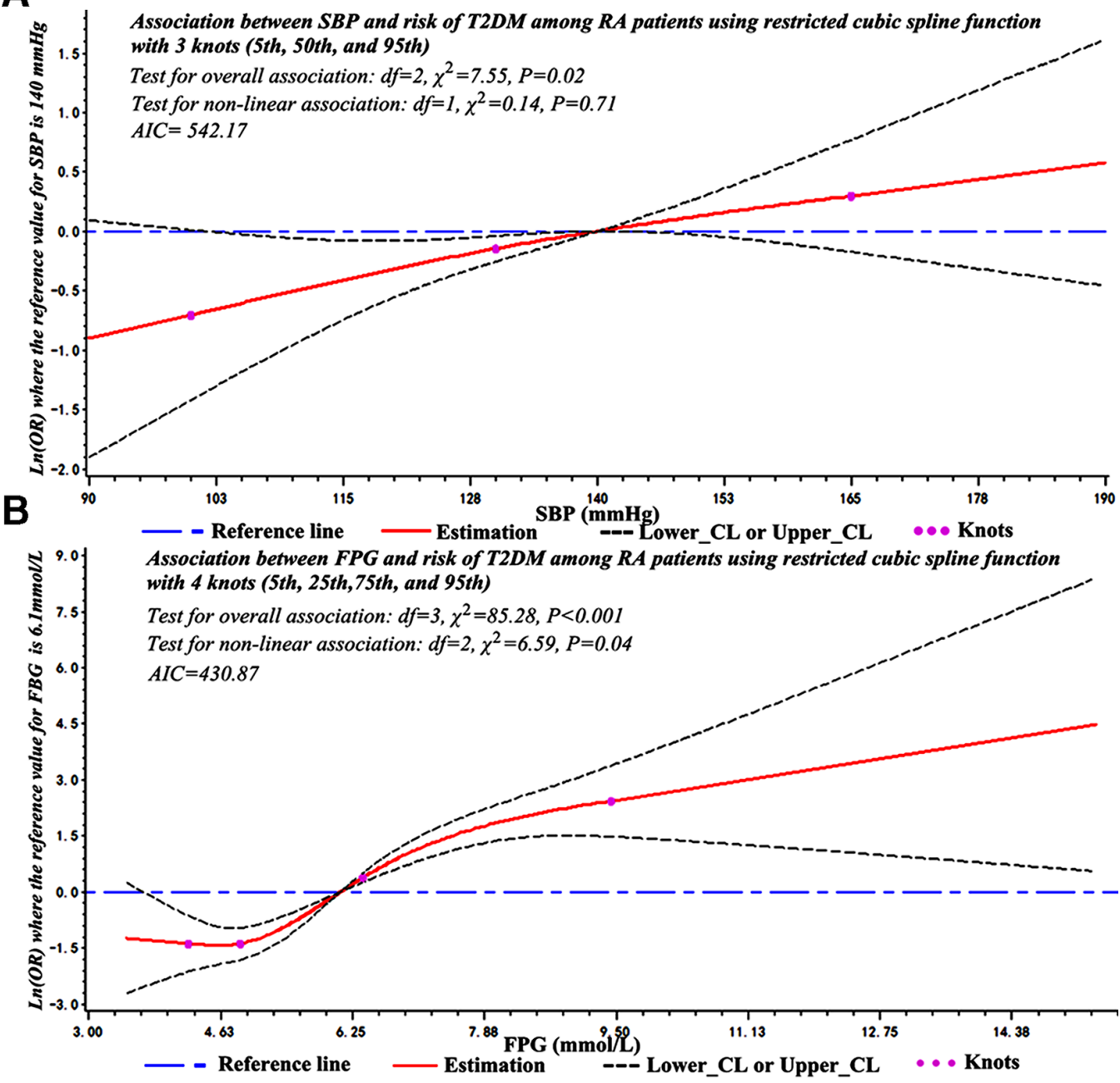

Figure 1 Adjusted dose-response association between SBP (A), FPG (B) and the risk of T2DM among patients with RA. Adjusted factors include age, sex, RF and/or IFG. The Y-axis indicates the In(OR) of T2DM for any value of SBP or FPG compared with the reference values. Dashed lines refer to the 95\% Cls. AIC, Akaike information criterion; FPG, fasting plasma glucose; IFG, impaired fasting glucose; RA, rheumatoid arthritis; RF, rheumatoid factor; SBP, systolic blood pressure; T2DM, type 2 diabetes mellitus.

risk of comorbid T2DM among patients with RA were linear, whereas the dose-response association between FPG and the risk of comorbid T2DM was non-linear.

Evidence has shown that RA and T2DM have a common pathological basis of inflammation. ${ }^{8}$ Patients with RA were more likely to develop CVD than the general population. ${ }^{9}$ Likewise, patients with T2DM were disproportionately affected by CVD when compared with the non-diabetic population. ${ }^{34}$ Therefore, there was no doubt that patients with RA with comorbid T2DM would bear a dramatically greater CVD disease burden than the healthy population. The majority of excess CVD risk is correlated to an increased prevalence of established traditional risk factors such as hypertension, hyperglycaemia and dyslipidaemia. $^{934}$

Our results showed that patients with RA with T2DM had higher SBP than patients with only RA. It was easy to predict the higher levels of FPG in patients with RA+T2DM than those in the RA only patients. In addition, a higher proportion of patients with RA+T2DM than RA only patients displayed IFG. As IFG is a transitional and reversible abnormality during the development of T2DM, this factor may theoretically represent a powerful but inexpensive tool for cardiometabolic risk stratification. ${ }^{35}$ IFG is superior to oral glucose tolerance in that the former could be easily identified with simple FPG measurements, but the latter needs to conduct an oral glucose tolerance test. Therefore, IFG was a good indicator of T2DM among patients with RA. Interestingly, the higher levels of SBP and FPG in patients with RA+T2DM might be partially attributed to therapeutic corticosteroid use, ${ }^{25}$ and this finding was further supported by the fact that the proportion of corticosteroid use in the RA+T2DM group was higher than that in the RA group $(76.82 \%$ vs $60.87 \%)$. Healthcare providers should carefully balance the benefits and risks before starting corticosteroid therapy. ${ }^{25}$ Interestingly, a recent study suggested a promising therapeutic choice for patients with RA+T2DM, interleukin (IL) $-1 \beta$ blocking molecules, as IL-1 $\beta$ is actively involved in the pathogenesis of both RA and T2DM. ${ }^{36}$ The authors 


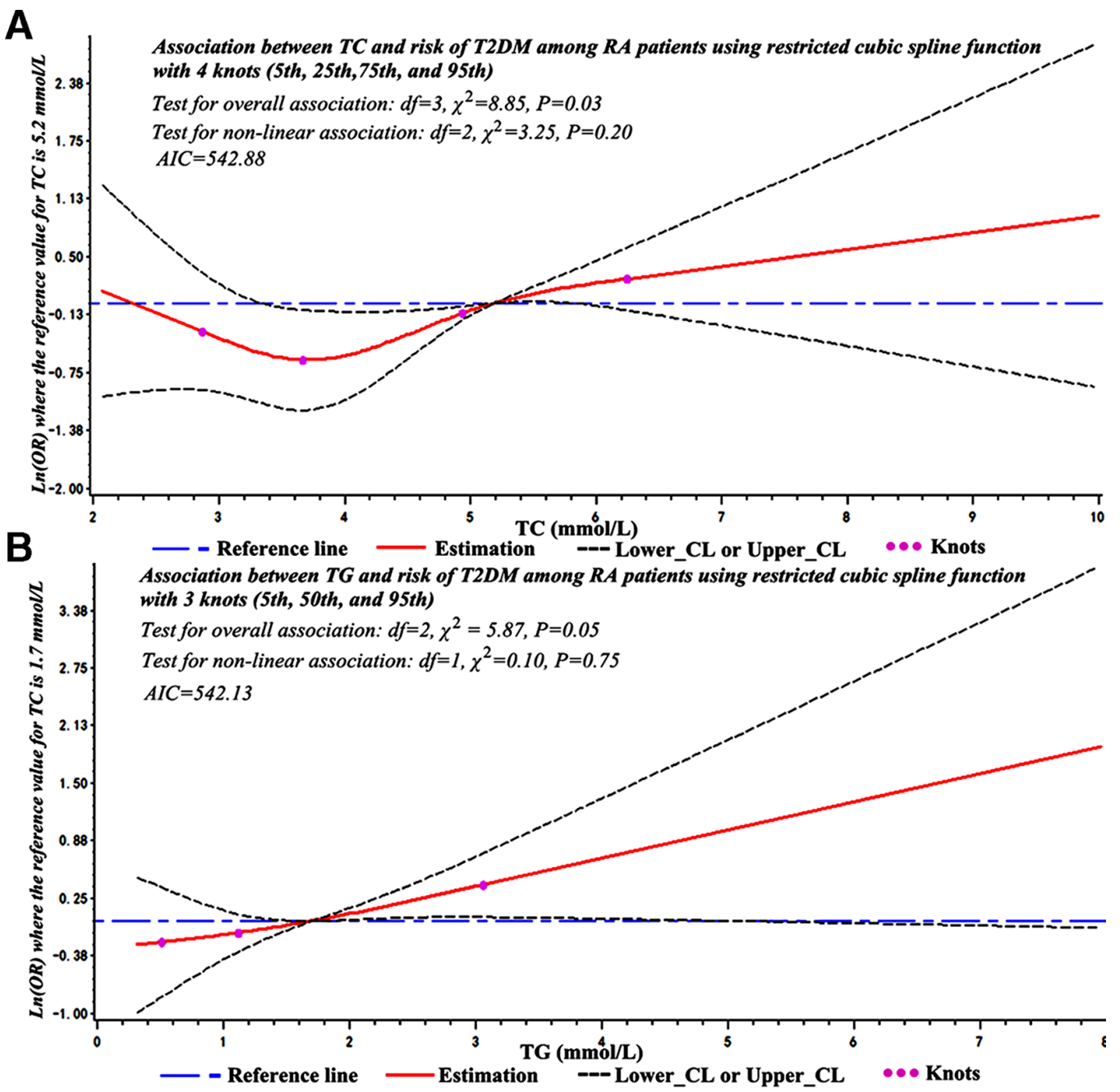

Figure 2 Adjusted dose-response association between TC (A), TG (B) and the risk of T2DM among patients with RA. Adjusted factors include age, sex, RF and IFG. The Y-axis indicates the In(OR) of T2DM for any value of TC or TG compared with the reference values. Dashed lines refer to the 95\% Cls. AIC, Akaike information criterion; IFG, impaired fasting glucose; RA, rheumatoid arthritis; T2DM, type 2 diabetes mellitus; TC, total cholesterol; TG, triglyceride.

found that active patients with RA with concomitant T2DM could achieve their treatment goals for both diseases (DAS28 $<2.6$ and HbA1c $<7 \%$ ) after taking anakinra. ${ }^{36}$ Evidence from another study showed that some non-tumour necrosis factor targeting biologics and small molecules (such as tocilizumab and abatacept) may have a role in improving insulin sensitivity. ${ }^{37}$ Under the setting of limited healthcare budgets, these targeted molecules with additional therapeutic effects for comorbidities are a strongly perceived need. ${ }^{38}$ Approximately $47.68 \%$ of our patients with RA+T2DM had an HbAlc level $>7 \%$, which indicated that almost half of the patients with RA+T2DM received insufficient antidiabetic treatment and that more aggressive glycaemia control was needed.

Compared with RA alone patients, those with RA+T2DM had higher levels of TG as well as higher proportions of TC and LDL-C dyslipidaemia. The lipid profiles of the two groups revealed that patients with RA+T2DM had a greater CVD disease burden than RA only patients. Among all types of lipid dyslipidaemia, HDL-C dyslipidaemia was the most common one, although the prevalence of HDL-C dyslipidaemia was not significantly different between the two groups. Our findings were consistent with previous studies. ${ }^{839}$ Surprisingly, we found that the DBP, FPG, HbA1c, TC, TG, HDL-C, LDL-C levels were lower in patients with RA+T2DM than in T2DM only patients. One reasonable explanation is that the DM duration of patients with RA+T2DM was much shorter than that of DM only patients (18 vs 78 months). A longer DM duration is related to a higher prevalence of CVD risk factors. Future studies should be conducted with newly diagnosed patients with T2DM to avoid potential bias.

The associations between SBP, FPG, TC, TG and the risk of comorbid T2DM among established patients with RA were further verified by the subsequent dose-response analyses. Importantly, using the drawn dose-response curves, we could calculate the risks of developing T2DM among patients with RA with any specific levels of the above indexes. This would facilitate T2DM risk assessments for patients with RA and enable individualised and tailored management strategies for patients with RA. 
As a retrospective observational study, some limitations could not be avoided. First, as the participants were recruited using EMRs and outpatients were not included, we were unable to calculate the exact incidence of T2DM in established patients with RA. Second, the data were retrospectively collected and a considerable proportion of participants were excluded because of missing values, which may lead to selection bias. Third, the confounding effects that the between-group treatment differences have on the various metabolic indexes should not be neglected, although age-PSM and sex-PSM was conducted. Fourth, as a case-control study without a follow-up period, the causal relationship between the potential factors and the risk of developing T2DM among patients with RA could not be definitively proven. Fifth, recent studies have revealed a close association between uncontrolled disease activity (poor EULAR-DAS28 response) and the risk of developing T2DM. ${ }^{40}$ However, we were unable to verify their findings as the EULAR-DAS28 forms were not routinely included in the medical records and the patients were not regularly followed up after discharge. Disease activity should be taken into consideration in future research. Finally, the single-centre design may reduce the external validity of our results. ${ }^{40}$ Nevertheless, our results reflect the current climate of managing of metabolic abnormalities among patients with RA with T2DM in a real-world clinical setting.

\section{CONCLUSIONS}

In conclusion, patients with RA+T2DM were more likely to exhibit metabolic abnormalities than those in RA only patients. Patients with RA+T2DM deserve more attention from rheumatologists and endocrinologists. Lipid levels should be routinely monitored and more stringent control of hypertension and glycaemia are warranted. Well-designed and large-sample studies are needed to validate our findings.

\section{Author affiliations}

${ }^{1}$ Department of Medical Record Management Center, The First Affiliated Hospital of China Medical University, Shenyang, China

${ }^{2}$ Department of Clinical Epidemiology and Evidence-Based Medicine, The First Affiliated Hospital of China Medical University, Shenyang, China

${ }^{3}$ Department of Experiment Teaching Center, School of Public Health, China Medical University, Shenyang, China

Acknowledgements The authors would like to thank Yiducloud (Beijing) Technology Ltd for their assistance in data extraction and processing.

Contributors LF and WC: conceived the study. GL and BB: performed the data collection. GL and TW: conducted the data analyses. GL and YL: drafted the manuscript. LF: took responsibility for the paper as a whole. All authors contributed substantially to the revision of the manuscript.

Funding This work was supported by grants from the Program of the National Natural Science Foundation of China (81673246).

Competing interests None declared.

Ethics approval The study protocol was approved by the ethics committee of the First Affiliated Hospital of China Medical University (research ethics board approval number 2016_48). This study was conducted following the principles of the 1964 Declaration of Helsinki.
Provenance and peer review Not commissioned; externally peer reviewed.

Data sharing statement Data can be made available upon request from readers.

Open access This is an open access article distributed in accordance with the Creative Commons Attribution Non Commercial (CC BY-NC 4.0) license, which permits others to distribute, remix, adapt, build upon this work non-commercially, and license their derivative works on different terms, provided the original work is properly cited, appropriate credit is given, any changes made indicated, and the use is non-commercial. See: http://creativecommons.org/licenses/by-nc/4.0/.

\section{REFERENCES}

1. Uhlig T, Moe RH, Kvien TK. The burden of disease in rheumatoid arthritis. Pharmacoeconomics 2014;32:841-51.

2. Cross M, Smith E, Hoy D, et al. The global burden of rheumatoid arthritis: estimates from the global burden of disease 2010 study. Ann Rheum Dis 2014;73:1316-22.

3. Hu H, Luan L, Yang K, et al. Burden of rheumatoid arthritis from a societal perspective: a prevalence-based study on cost of this illness for patients in China. Int J Rheum Dis 2018;21:1572-80.

4. Fu L, Zhang J, Jin L, et al. A case-control study of rheumatoid arthritis revealed abdominal obesity and environmental risk factor interactions in northern China. Mod Rheumatol 2018;28:249-57.

5. Dougados M, Soubrier M, Antunez A, et al. Prevalence of comorbidities in rheumatoid arthritis and evaluation of their monitoring: results of an international, cross-sectional study (COMORA). Ann Rheum Dis 2014;73:62-8.

6. Radner H, Yoshida K, Hmamouchi I, et al. Treatment patterns of multimorbid patients with rheumatoid arthritis: results from an international cross-sectional Study. J Rheumatol 2015;42:1099-104.

7. Joshi AA, Lerman J, Aberra T, et al. Increased clinical and financial burden of heart failure hospitalizations in patients with rheumatoid arthritis: Insights from the national inpatient sample. J Am Coll Cardiol 2018;71:A903-03.

8. Liu XZ, Gao Y, Fan J, et al. Metabolic abnormalities in rheumatoid arthritis patients with comorbid diabetes mellitus. Clin Rheumatol 2018;37:219-26.

9. Puttevils D, De Vusser P, Geusens P, et al. Increased cardiovascular risk in patients with rheumatoid arthritis: an overview. Acta Cardiol 2014;69:111-8.

10. Lindhardsen J, Ahlehoff $\mathrm{O}$, Gislason $\mathrm{GH}$, et al. The risk of myocardial infarction in rheumatoid arthritis and diabetes mellitus: a Danish nationwide cohort study. Ann Rheum Dis 2011;70:929-34.

11. van Halm VP, Peters MJ, Voskuyl AE, et al. Rheumatoid arthritis versus diabetes as a risk factor for cardiovascular disease: a cross-sectional study, the CARRE Investigation. Ann Rheum Dis 2009;68:1395-400.

12. Ursini F, D Angelo S, Russo E, et al. Serum Complement C3 and Type 2 Diabetes in rheumatoid arthritis: a case-control study. Rev Recent Clin Trials 2018;13:215-21.

13. Liu X, Li Y, Li L, et al. Prevalence, awareness, treatment, control of type 2 diabetes mellitus and risk factors in Chinese rural population: the RuralDiab study. Sci Rep 2016;6:31426.

14. Yan L, Xu MT, Yuan L, et al. Prevalence of dyslipidemia and its control in type 2 diabetes: a multicenter study in endocrinology clinics of China. J Clin Lipidol 2016;10:150-60.

15. Ji L, Zhi X, Lu J, et al. Hyperglycemia and blood pressure treatment goal: a cross sectional survey of 18350 patients with type 2 diabetes in 77 tertiary hospitals in China. PLoS One 2014;9:e103507.

16. Bartels CM, Kind AJ, Thorpe CT, et al. Lipid testing in patients with rheumatoid arthritis and key cardiovascular-related comorbidities: a medicare analysis. Semin Arthritis Rheum 2012;42:9-16.

17. Laird-Maddox M, Mitchell SB, Hoffman M. Integrating research data capture into the electronic health record workflow: real-world experience to advance innovation. Perspect Health Inf Manag 2014;11:1e.

18. Kamal KM, Chopra I, Elliott JP, et al. Use of electronic medical records for clinical research in the management of type 2 diabetes. Res Social Adm Pharm 2014;10:877-84.

19. Liao KP, Cai T, Gainer V, et al. Electronic medical records for discovery research in rheumatoid arthritis. Arthritis Care Res 2010;62:1120-7.

20. Baek S, Park SH, Won E, et al. Propensity score matching: a conceptual review for radiology researchers. Korean J Radiol 2015;16:286-96.

21. Aletaha D, Neogi T, Silman AJ, et al. Rheumatoid arthritis classification criteria: an American College of Rheumatology/ European League Against Rheumatism collaborative initiative. Arthritis Rheum 2010;2010:2569-81. 
22. Arnett FC, Edworthy SM, Bloch DA, et al. The American Rheumatism Association 1987 revised criteria for the classification of rheumatoid arthritis. Arthritis Rheum 1988;31:315-24.

23. Graf J, Scherzer R, Grunfeld C, et al. Levels of C-reactive protein associated with high and very high cardiovascular risk are prevalent in patients with rheumatoid arthritis. PLoS One 2009;4:e6242.

24. Go DJ, Lee EY, Lee EB, et al. Elevated erythrocyte sedimentation rate is predictive of interstitial lung disease and mortality in dermatomyositis: a Korean Retrospective Cohort Study. J Korean Med Sci 2016;31:389-96.

25. Strehl C, Bijlsma JW, de Wit M, et al. Defining conditions where longterm glucocorticoid treatment has an acceptably low level of harm to facilitate implementation of existing recommendations: viewpoints from an EULAR task force. Ann Rheum Dis 2016;75:952-7.

26. McLaughlin T, Reaven G, Abbasi F, et al. Is there a simple way to identify insulin-resistant individuals at increased risk of cardiovascular disease? Am J Cardiol 2005;96:399-404.

27. Hoyo C, Cook MB, Kamangar F, et al. Body mass index in relation to oesophageal and oesophagogastric junction adenocarcinomas: a pooled analysis from the International BEACON Consortium. Int $J$ Epidemiol 2012;41:1706-18.

28. Desquilbet L, Mariotti F. Dose-response analyses using restricted cubic spline functions in public health research. Stat Med 2010;29:1037-57.

29. Li G, Qi G, Zhang B, et al. The dose-response association between estimated glomerular filtration rate and prognosis of patients with STsegment elevation myocardial infarction from rural areas of China's Liaoning province. Medicine 2017;96:e9508.

30. Thoemmes F. Propensity score matching in SPSS. Statistics 2012.

31. Austin PC. A comparison of 12 algorithms for matching on the propensity score. Stat Med 2014;33:1057-69.
32. Kang KY, Kim YK, Yi H, et al. Metformin downregulates Th17 cells differentiation and attenuates murine autoimmune arthritis. Int Immunopharmacol 2013;16:85-92.

33. Mahmood K, Naeem M, Rahimnajjad NA. Metformin: the hidden chronicles of a magic drug. Eur J Intern Med 2013;24:20-6.

34. Martín-Timón I, Sevillano-Collantes C, Segura-Galindo A, et al. Type 2 diabetes and cardiovascular disease: Have all risk factors the same strength? World J Diabetes 2014;5:444-70.

35. Ruscitti P, Ursini F, Cipriani P, et al. Prevalence of type 2 diabetes and impaired fasting glucose in patients affected by rheumatoid arthritis: Results from a cross-sectional study. Medicine 2017;96:e7896.

36. Giacomelli R, Ruscitti P, Alvaro S, et al. IL-1 $\beta$ at the crossroad between rheumatoid arthritis and type 2 diabetes: may we kill two birds with one stone? Expert Rev Clin Immunol 2016;12:849-55.

37. Ursini F, Russo E, Ruscitti P, et al. The effect of non-TNF-targeted biologics and small molecules on insulin resistance in inflammatory arthritis. Autoimmun Rev 2018:17:399-404.

38. Osiri M, Sattayasomboon Y. Prevalence and out-patient medical costs of comorbid conditions in patients with rheumatoid arthritis. Joint Bone Spine 2013;80:608-12.

39. Erum U, Ahsan T, Khowaja D. Lipid abnormalities in patients with Rheumatoid Arthritis. Pak J Med Sci 2017;33:227-30.

40. Ruscitti P, Ursini F, Cipriani P, et al. Poor clinical response in rheumatoid arthritis is the main risk factor for diabetes development in the short-term: A 1-year, single-centre, longitudinal study. PLoS One 2017;12:e0181203.

41. Crepaldi G, Scirè CA, Carrara G, et al. Cardiovascular comorbidities relate more than others with disease activity in rheumatoid arthritis. PLoS One 2016;11:e0146991. 